\title{
A SEARCH FOR OPTICAL COUTERPARTS OF GLOBULAR CLUSTER X-RAY SOURCES
}

\author{
Michel Aurière \\ Lydie Koch-Miramond \\ European Southern Obs. \\ Center of Nuclear Studies \\ Claude Chevalier \\ Jean-Pierre Cordoni \\ Sergio Ilovaisky \\ Obs. of Haute-Provence \\ University of Montpellier
}

M 15:AC211/4U2131+11: THE OPTICALLY IDENTIFIED X-RAY SOURCE IN M 15

The identification of the optical counterpart of the M 15 (NGC 7078) X-ray source has been confirmed photometrically and spectroscopically (Aurière et al. 1984, 1985, 1986; Ilovaisky and Chevalier: 1985; Charles et al. 1985, 1986). It is designated M 15:AC211 from the Aurière and Cordoni catalogue (1981).

Rapid large amplitude brightness variations of M15:AC211 in the U passband were discovered one year ago (Aurière et al. 1985; Ilovaisky and Chevalier 1985). These variations are very likely to be periodic but it turned out to be impossible to derive without ambiguity the period from series of data obtained at one site at once. We got recently new series of observations using both the $2 \mathrm{~m}$ telescope of Pic du Midi Observatory and the 3.6m Canada-France-Hawaii telescope on the same nights. These new data are under reduction.

Averaged magnitude and colours for M 15:AC211 when bright are: $\mathrm{U}=14.6, \mathrm{U}-\mathrm{B}=-1.2, \mathrm{~B}-\mathrm{V}=-0.1$, all \pm 0.2 . Taking into account EXOSAT measurements (courtesy M. Redfern) we find a ratio $\mathrm{L}_{X} / \mathrm{L}_{\text {opt }}$ of about 20 (X: $2 \mathrm{kev}-11 \mathrm{kev;}$ opt: $3000 \AA-7000 \AA)$. Both this low value and the large optical variations suggest that $M 15: A C 211 / 4 U 2131+11$ is a binary system observed at high inclination like Her X1, 0921-63, 1822-37, 2129+47. This gives a natural explanation for the M $15 \mathrm{X}$-ray source being the only globular cluster source which has never been observed to burst: the main part of the X-ray emission could be shielded by the accretion disk; it is thus possible that the $X$-ray source is a very high luminosity non-bursting one. Obtaining the period and the exact shape of the brightness variations will enable one to know the nature of the companion (main sequence or post m.s. star?) and the nature of the variable UV emission (bright accretion disk as in 1822-37?; heated face of the companion as in $2129+47$ ?).

683

J. E. Grindlay and A. G. Davis Philip (eds.),

The Harlow-Shapley Symposium on Globular Cluster Systems in Galaxies, 683-684.

(C) 1988 by the IAU. 
We have made recently a reinvestigation of the HRI Einstein satellite error boxes (Grindlay et al. 1984) for some of the southern $\mathrm{X}$-ray globular clusters. We used a UV coated GEC CCD (Cullum et al. 1985 ) at the $2.2 \mathrm{~m}$ telescope of La Silla (ESO). UBV images in good seeing conditions (FWHM of stellar profiles $0: 8$ in $\mathrm{V}, 1$ " in U) were obtained for 47 TUC, NGC 1851, NGC 6441, NGC 6624. They are now being reduced. A quick look examination showed that no object as prominent as M 15:AC211 was observed in the error boxes for the X-ray sources.

Two most interesting targets were NGC 1851 and NGC 6624 because a possible UV counterpart could have been observed with IUE (Grindlay 1983).

We present our provisional result for NGC 1851: 5 stars are resolved nearer than $3 "$ from the HRI Einstein position for the X-ray source. None of them is either hot enough to explain the UV emission or variable. On the other hand, blue horizontal branch stars which could be responsible for the UV emission are found in the field.

Thus, the optical counterpart of the X-ray source in NGC 1851 does not appear to be detected. This suggests that, if this object is a neutron star binary, the companion is unlikely to be a heated post main-sequence star as could be the case for M.15 (Aurière et al. 1986).

\section{REFERENCES}

Aurière, M. and Cordoni, J. P. 1981 Astron. Astrophys. Supp1. 46,347 .

Aurière, M., Le Fèvre, 0 and Terzan, A. 1984 Astron. Astrophys. $138,415$.

Aurière, M., Cordoni, J. P. and Koch-Miramond, L. 1985 IAUC 4101.

Aurière, M., Maucherat, A., Cordoni, J. P., Fort, B. and Picat, J. P. 1986 Astron. Astrophys. 158, 158 .

Charles, P. A., Aurière, M., Ilovaisky, S. and Koch-Miramond, L. 1985 IAUC 4146 .

Charles, P. A., Jones, D. C. and Naylor, T. Nature, submitted.

Cullum, M. Deireies, S., D'Odorico, S. and Reiss, R. 1985 Astron. Astrophys. 153, L1.

Grindlay, J. E. 1983 Advances in Space Research 2, No. 9 p. 133.

Grindlay, J. E., Hertz, P. Steiner, J. E., Murray, S. S., and Lightman, A. P. 1984 Astrophys. J. Letters 282, L13.

Ilovaisky, S. and Chevalier, C. 1985 IAUC 4146. 